\title{
Anti-C1q autoantibodies amplify pathogenic complement activation in systemic lupus erythematosus
}

\author{
V. Michael Holers
}

Departments of Medicine and Immunology, University of Colorado Health Sciences Center, Denver, Colorado, USA.

\begin{abstract}
Patients with systemic lupus erythematosus (SLE) often develop glomerulonephritis (i.e., inflammation in the glomeruli of the kidney), commonly referred to as lupus nephritis. Patients with lupus nephritis typically have autoantibodies to the complement classical pathway protein C1q. Whether these anti-C1q antibodies play any role in the development of lupus nephritis has been unclear. In this issue of the JCI, a new study demonstrates that anti-C1q antibodies can amplify glomerular injury but only when they are bound within the glomerulus to $\mathrm{C} 1 \mathrm{q}$ that has been already brought to that site by other types of glomerular-reactive autoantibodies (see the related article beginning on page 679). These studies are the first, to our knowledge, to provide a causal link between anti-C1q antibodies and target organ damage in SLE.
\end{abstract}

The complement system is a central component of innate immunity that exhibits three pathways of activation: classical, alternative, and lectin-mediated. C1, a key component of the classical pathway, is actually a complex of three proteins: $\mathrm{C} 1 \mathrm{q}, \mathrm{C} 1 \mathrm{r}$, and C1s (1). C1q is a collagen-like component that is able to bind antibodies but only after the antibody has been bound to a foreign or self antigen. Once $\mathrm{C} 1 \mathrm{q}$ is bound to the $\mathrm{Fc}$ antibody domain, $\mathrm{C} 1 \mathrm{r}$ and $\mathrm{C} 1 \mathrm{~s}$ are sequentially cleaved and released, after which the rest of the classical pathway is activated. Immune complexes normally contain $\mathrm{C1q}$ bound via its "head" domains to Fc regions of $\operatorname{IgG}$ as part of the activation function of C1q within the classical pathway (1) (Figure 1). An alternate means of binding $\mathrm{C} 1 \mathrm{q}$, though, has also been described; it occurs when high-affinity autoantibodies directly recognize the collagenous "tail" portion of $\mathrm{C} 1 \mathrm{q}$ through the antibody $\mathrm{F}(\mathrm{ab})$ antigen-combining sites rather than via the Fc domain. Since they were first described $(2,3)$, anti-C1q autoantibodies have been commonly identified in patients with autoimmune diseases such as systemic lupus erythematosus (SLE) and hypocomple-

Nonstandard abbreviations used: MAC, membrane attack complex; SLE, systemic lupus erythematosus.

Conflict of interest: The author has declared that no conflict of interest exists.

Citation for this article: J. Clin. Invest. 114:616-619 (2004). doi:10.1172/JCI200422820. mentemic urticarial vasculitis. Although anti-C1q antibodies are associated with the presence of lupus nephritis - indeed probably serving as a biomarker for the presence of renal disease (4) - and antiC1q antibodies are also preferentially localized in the glomeruli of patients with SLE (5), their pathophysiologic importance has remained undefined. Specifically, whether this class of acquired autoantibodies is merely an epiphenomenon or is truly pathogenic, and if so how and under what clinical circumstances, has remained an unanswered question.

\section{Anti-C1q autoantibodies are pathogenic}

In this issue of the JCI, Trouw et al. (6) have now solved an important piece of this puzzle by first developing a murine $\mathrm{mAb}$, JL-1, which was identified by ELISA based on its ability to recognize the tail domain of mouse C1q. When anti-C1q JL-1 was administered alone, it was bound in the glomerulus to $\mathrm{C} 1 \mathrm{q}$, which is normally present there at low levels; however, this interaction was insufficient to induce significant glomerular damage (Figure 1A). However, when JL-1 was administered to mice in which C1q levels in the glomerulus were greatly elevated as a consequence of its interaction with other antibodies with specificity for glomerular antigens, mice then exhibited significant glomerular injury as shown by decreased renal function and elevated "leakage" of protein into the urine (6) (Figure 1B).

The combination of the first glomerularbinding antibody and JL-1 caused glomerular injury in a complement C4-, C3-, and Fc-dependent manner, reflecting a key role of the classical pathway itself in the generation of $\mathrm{C} 3 \mathrm{a}, \mathrm{C} 5 \mathrm{a}$, and the membrane attack complex (MAC). These downstream complement activation fragments are key mediators of complement-catalyzed autoimmune renal injury (7) (Figure 1B). In the setting described by Trouw et al., these complement mediators were probably generated by both types of antibodies, the initial glomerular-targeting antibodies as well as mAb JL-1. Together, the two types of antibodies generated enough mediators to be clinically important and cause glomerular injury in vivo. What do these results tell us about the role of $\mathrm{C} 1 \mathrm{q}$ in SLE and also about this intriguing class of acquired autoantibodies?

First, one has to ask whether the lone monoclonal antibody, JL-1, utilized in this study (6) to amplify glomerular injury is representative of the polyclonal population of C1q-reactive antibodies in human patients. It could be argued, as is well known in murine models, that placement of a "planted antigen" (herein possibly C1q) in the glomerulus followed by administration of a complement-fixing antibody that targets the antigen in situ readily leads to complement-dependent injury (8). The model system utilized by Trouw et al. (6) simply recapitulates this phenotype but in a clinically unrelated fashion. In addition, as pointed out by the authors, previous experiments in mice using glomerulartargeting antibodies also demonstrate dose-dependent "windows," in which the injurious effects of complement activation are more prominent than at higher or lower doses of antibody (9). In this light, the use by the authors of a broad range of doses (of each reagent, the C1q-fixing anti-glomerular basement membrane anti- 
A
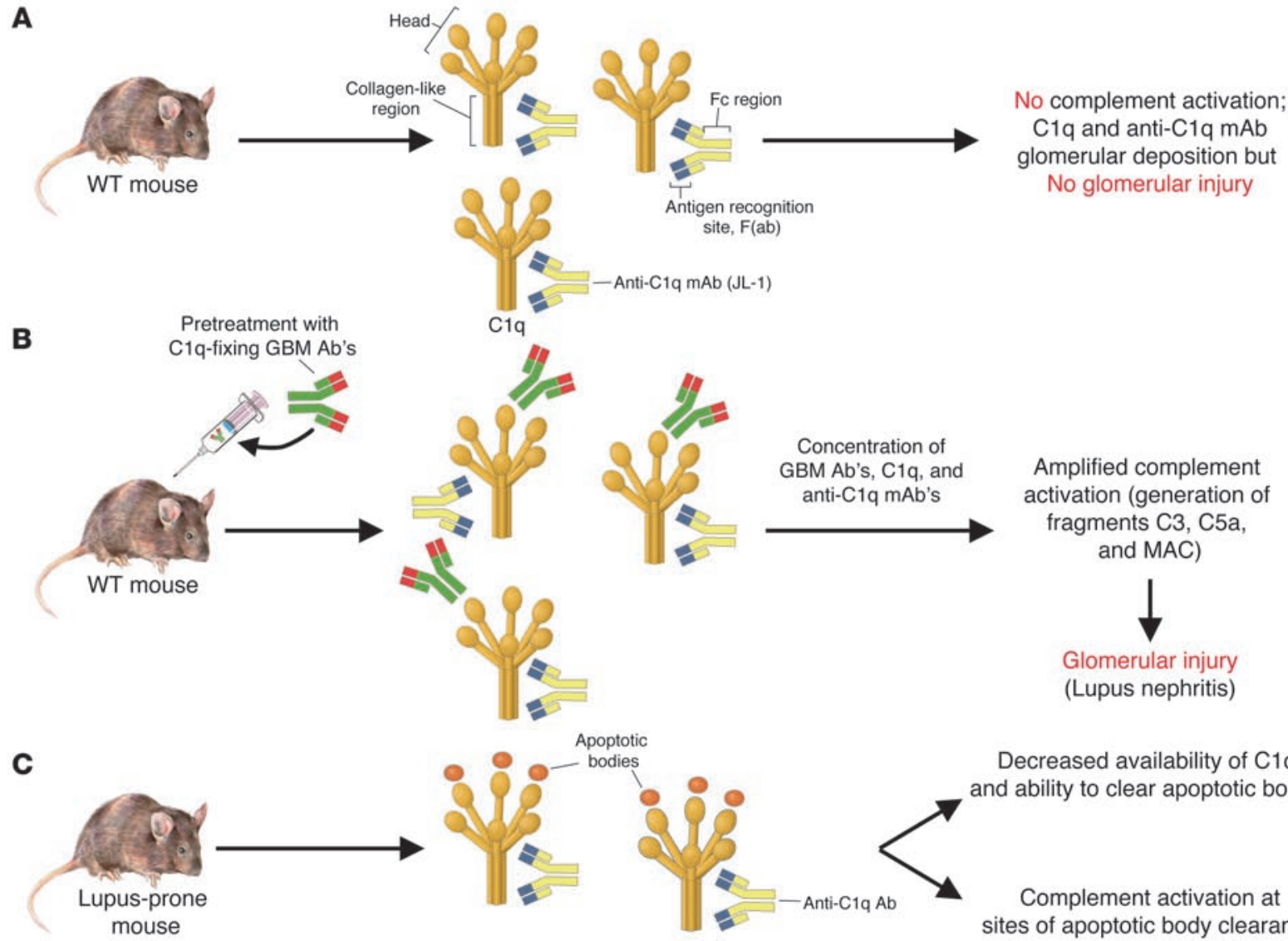

Amplified complement activation (generation of fragments $\mathrm{C} 3, \mathrm{C} 5 \mathrm{a}$, and $\mathrm{MAC}$ )

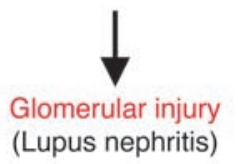

Glomerular injury
(Lupus nephritis)

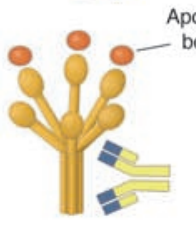

Apoptotic
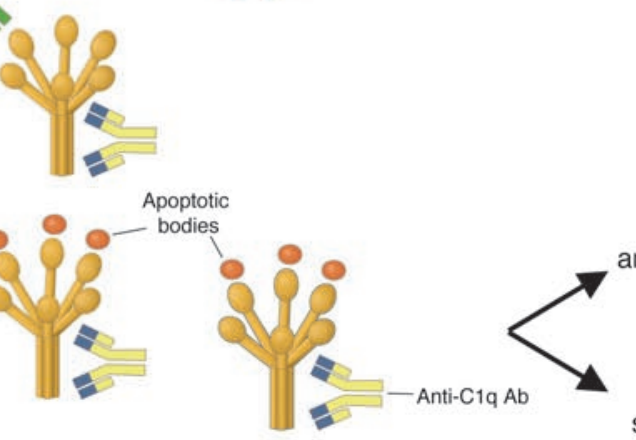

Decreased availability of C1q and ability to clear apoptotic bodies

Complement activation at sites of apoptotic body clearance

\section{Figure 1}

Roles of anti-C1q antibodies in the development of glomerular injury and antinuclear antibodies. (A) Anti-C1q antibodies (in yellow) such as JL-1 recognize the collagen-like "tails" of $\mathrm{C} 1 \mathrm{q}$ in much the same manner as they would recognize any antigen through the $\mathrm{F}(\mathrm{ab})$ antigen-recognition domain. The administration of $\mathrm{C} 1 \mathrm{q}$ and anti-C1q is not sufficient to cause glomerular injury as shown by Trouw et al. (6). However, when $\mathrm{C} 1 \mathrm{q}-$ fixing anti-glomerular basement membrane (GBM) antibodies (in green) are first administered to mice, then C1q is able to bind to the Fc domain as it normally does. This brings anti-C1q antibodies into the glomerulus, resulting in sufficient complement activation to result in the generation of $\mathrm{C} 3 \mathrm{a}, \mathrm{C} 5 \mathrm{a}$, and MAC and the development of glomerulonephritis (B). As an alternate means by which anti-C1q antibodies could promote lupus-like autoimmunity, these antibodies could interfere with the normal ability of $\mathrm{C} 1 \mathrm{q}$ to recognize apoptotic bodies containing DNA and other nuclear autoantigens $(\mathbf{C})$. In this scenario, impaired clearance of apoptotic bodies, or clearance in a proinflammatory setting due to complement activation caused by the anti-C1q antibodies, could promote the development of autoantibodies that target DNA and other nuclear antigens, which is similar to what occurs when $\mathrm{C} 1 \mathrm{q}$ is absent due to a genetic deficiency.

body, and JL-1) would show how narrow the effect of the addition of monoclonal anti-C1q antibody on the development of glomerular injury is.

However, in support of a close relationship between these findings in mice and SLE-associated lupus nephritis in humans, $\mathrm{JL}-1$ is reported to recognize the same collagen-like domain of $\mathrm{C} 1 \mathrm{q}$ as do human antiC1q antibodies $(2,3,6)$. In addition, previous studies in which $\mathrm{C} 1 \mathrm{q}$ and polyclonal anti-C1q antibodies were both transferred into mice resulted in glomerular targeting of anti-C1q antibodies (10) as well as modest glomerular damage (11) similar to that caused by mAb JL-1 alone in the study by Trouw et al. (6). Nevertheless, a stronger link with human disease may be provided by a more careful comparison of the specific epitope reactivity of JL-1 and authentic autoantibodies from patients with glomerulonephritis. For example, is there evidence of cross-competition for $\mathrm{C} 1 \mathrm{q}$ epitopes between human polyclonal anti-C1q autoantibodies and JL-1?

\section{Anti-C1q antibodies increase complement activation in a relatively uncontrolled fashion}

The complement system itself is regulated positively by amplification mechanisms (12) and negatively by regulatory proteins (13). At each activation step, a small amount of activated product can lead to the generation of from four to several thousand activated components derived from the imme- diate downstream target (1). The alternative pathway demonstrates an "amplification loop" effect, where C3b generated from the classical pathway can serve to bind factor B and initiate further $\mathrm{C} 3$ activation through formation of the $\mathrm{C} 3$ convertase $\mathrm{C} 3 \mathrm{bBb}$ (12) (Figure 2). Although often thought of as a minor contributor to total complement activation - which is true if one considers only serum activation - amplification of injury in a target organ through engagement of the alternative pathway, amplifying injury in a target organ, is absolutely essential to the generation of local C5a- and MAC-dependent injury $(14,15)$.

This concept is relevant to anti-C1q antibodies because the studies of Trouw et al. (6) strongly suggest that these 


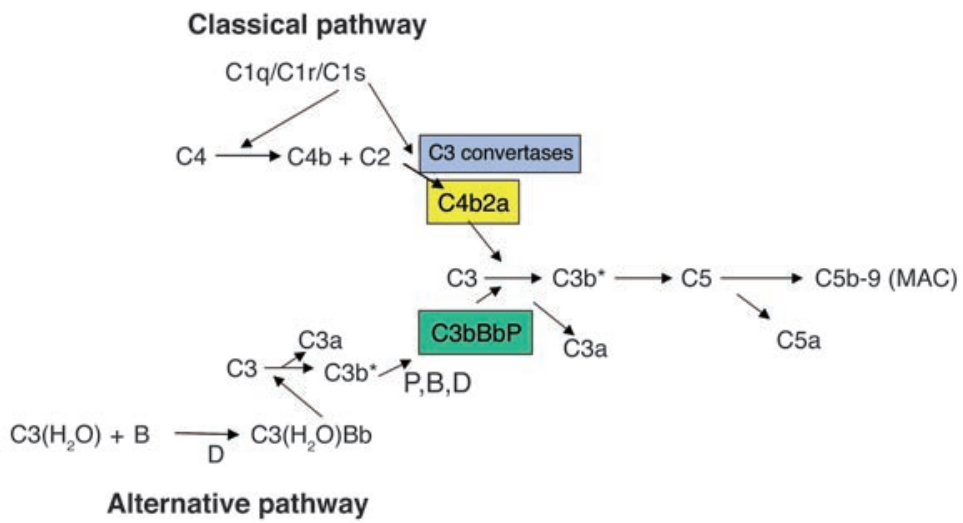

Figure 2

Simplified schematic demonstrating mechanisms of activation of classical and alternative pathways and generation of C3 convertases (light blue). The alternative pathway C3 convertase (green box) can be generated by the activity of the classical pathway C3 convertase C4b2a (yellow box) on $\mathrm{C} 3$, which results in $\mathrm{C} 3 \mathrm{~b}$ formation. This is called the alternative pathway amplification loop. In patients with C4 nephritic factors, autoantibodies react with the complex of $\mathrm{C} 4 \mathrm{~b} 2 \mathrm{a}$ and keep it from being inactivated, thus generating more C3b than would normally occur. $\mathrm{C}^{*} \mathrm{~b}^{*}$ in the alternative pathway can originate from C3b generated by the classical pathway C3 convertase C4b2a.

autoantibodies likewise serve as an acquired mechanism of classical pathway amplification. Previously, the only means to amplify the classical pathway beyond what is possible through endogenous classical pathway components has been with C4-nephritic factor. This type of autoantibody, occasionally found in patients, stabilizes the classical pathway C3 convertase C4b2a and allows this convertase to generate far more activated C3 molecules than it normally would (16). Trouw et al. demonstrate that anti-C1q autoantibodies can result in a similarly amplified biologic effect of complement in vivo locally in the kidney, presumably by generating additional C3 through the classical pathway. In this light, it would be of some interest to determine the exact mechanism by which the classical pathway is amplified by JL-1 and whether this antibody interferes with other classical pathway regulatory mechanisms.

\section{Additional deleterious roles potentially played by anti-C1q autoantibodies}

In the larger context of lupus-like autoimmunity, C1q has taken on an increasingly important role and is necessary not only for classical pathway-dependent complement activation in target organs, as focused upon by Trouw et al. in this issue (6), but is also required to directly recognize and help to clear potentially dangerous nuclear autoantigens from apoptotic cells (17). Thus, in patients (18) and in certain auto- immune mouse strains (19), the absence of C1q leads to the development of anti-DNA antibodies and SLE. Of interest, C1q-deficient patients commonly exhibit severe renal disease (18), the cause of which has been ascribed to non-complement-dependent mechanisms, as C3 is not required in mice to develop glomerular injury in the absence of C1q (20).

In this context of multiple roles for $\mathrm{C} 1 \mathrm{q}$, one could hypothesize that anti-C1q autoantibodies not only affect patients with SLE by injuring the kidney, as suggested by Trouw et al. (6), but also by enhancing the development of anti-DNA and other glomerular-targeting nuclear autoantibodies, because there is too little C1q available for effective clearance of these autoantibodies would not only amplify local injury but also potentially accelerate the development of antinuclear autoantibodies by interfering with $\mathrm{C} 1 \mathrm{q}$ clearance functions (21). Alternatively, if these autoantibodies also lead to enhanced complement activation at sites where $\mathrm{C} 1 \mathrm{q}$ is recognizing nuclear antigens, this could in principle switch noninflammatory recognition of apoptotic bodies by $\mathrm{C} 1 \mathrm{q}$ and its receptors to inflammatory recognition when $\mathrm{C} 5 \mathrm{a}$ and other complement activation fragments are also generated, and their receptors are engaged on cells clearing these antigens.

In sum, acquired anti-C1q autoantibodies could utilize several possible mechanisms by which they could increase the dangerous antigens (Figure 1C). Thus, these severity of an autoimmune response and glomerulonephritis. The studies by Trouw et al. (6) provide an important conceptual advance in this area and open up the possibility of determining how inhibiting C1q or modulating its effects leads to severe SLE. In particular, the use of JL- 1 and similar monoclonal antibodies in mouse models should allow these and other investigators to better understand the molecular mechanisms that lead both to increased development of antiDNA antibodies and to tissue injury.

Address correspondence to: V. Michael Holers, Departments of Medicine and Immunology, Box B-115, University of Colorado Health Sciences Center, 4200 East Ninth Avenue, Denver, Colorado 80262, USA. Phone: (303) 315-7592; Fax: (303) 3155540; E-mail: michael.holers@uchsc.edu.

1. Lachmann, P.J., and Hughes-Jones, N.C. 1984. Initiation of complement activation. Springer Semin. Immunopathol. 7:143-162

2. Uwatoko, S., and Mannik, M. 1988. Low-molecular weight $\mathrm{C} 1 \mathrm{q}$-binding immunoglobulin $\mathrm{G}$ in patients with systemic lupus erythematosus consists of autoantibodies to the collagen-like region of C1q. J. Clin. Invest. 82:816-824.

3. Wisnieski, J.J., and Naff, G.B. 1989. Serum IgG antibodies to $\mathrm{C} 1 \mathrm{q}$ in hypocomplementemic urticarial vasculitis syndrome. Arthritis Rheum. 32:1119-1127.

4. Coremans, I.E.M., et al. 1995. Changes in antibodies to $\mathrm{C} 1 \mathrm{q}$ predict renal relapses in systemic lupus erythematosus. Am. J. Kidney Dis. 26:595-601.

5. Mannik, M., and Wener, M.H. 1997. Deposition of antibodies to the collagen-like region of $\mathrm{C} 1 \mathrm{q}$ in renal glomeruli of patients with proliferative lupus glomerulonephritis. Arthritis Rheum. 40:1504-1511.

6. Trouw, L.A., et al. 2004. Anti-C1q autoantibodies deposit in glomeruli but are only pathogenic in combination with glomerular C1q-containing immune complexes. J. Clin. Invest. 114:679-688. doi:10.1172/JCI200421075.

7. Quigg, R.J. 2004. Complement and autoimmune glomerular diseases. Curr. Dir. Autoimmun. 7:165-180.

8. Feintzeig, I.D., Dittmer, J.E., Cybulski, A.V., and Salant, D.J. 1986. Antibody, antigen, and glomerular capillary wall charge interactions: influence of antigen location on in situ immune complex formation. Kidney Int. 29:649-657.

9. Quigg, R.J., et al. 1998. Blockade of antibodyinduced glomerulonephritis with Crry-Ig, a soluble murine complement inhibitor. J. Immunol. 160:4553-4560.

10. Uwatoko, S., Gauthier, V.J., and Mannik, M. 1991. Autoantibodies to the collagen-like region of C1Q deposit in glomeruli via C1Q in immune deposits. Clin. Immunol. Immunopathol. 61:268-273.

11. Trouw, L.A., et al. 2003. Glomerular deposition of $\mathrm{C} 1 \mathrm{q}$ and anti-C1q antibodies in mice following injection of antimouse C1q antibodies. Clin. Exp. Immunol. 132:32-39.

12. Muller-Eberhard, H.J. 1988. Molecular organization and function of the complement system. Ann. Rev. Biochem. 57:321-347.

13. Liszewski, M.K., Farries, T.C., Lublin, D.M., Rooney, I.A., and Atkinson, J.P. 1996. Control of the complement system. Adv. Immunol. 61:201-283.

14. Girardi, G., et al. 2003. Complement C5a receptors and neutrophils mediate fetal injury in 
the antiphospholipid syndrome. J. Clin. Invest. 112:1644-1654. doi:10.1172/JCI200318817.

15. Holers, V.M., and Thurman, J.M. 2004. The alternative pathway of complement in disease: opportunities for therapeutic targeting. Mol. Immunol. 41:147-152.

16. Gigli, I., Sorvillo, J., Mecarelli-Halbwachs, L., and Leibowitch, J. 1981. Mechanism of action of the
C4 nephritic factor. Deregulation of the classical pathway of C3 convertase. J. Exp. Med. 154:1-12.

17. Botto, M., et al. 1998. Homozygous C1q deficiency causes glomerulonephritis associated with multiple apoptotic bodies. Nat. Genet. 19:56-59.

18. Bowness, P., et al. 1994. Hereditary C1q deficiency and systemic lupus erythematosus. Q. J. Med. 87:455-464.
19. Mitchell, D.A., et al. 2002. C1q deficiency and autoimmunity: the effects of genetic background on disease expression. J. Immunol. 168:2538-2543.

20. Mitchell, D.A., et al. 1999. C1q protects against the development of glomerulonephritis independently of C3 activation. J. Immunol. 162:5676-5679.

21. Botto, M., and Walport, M.J. 2002. C1q, autoimmunity and apoptosis. Immunobiology. 205:395-406.

\title{
Stat3 is required for the development of skin cancer
}

\author{
Laura Pedranzini, Andrea Leitch, and Jacqueline Bromberg
}

Memorial Sloan Kettering Cancer Center, New York, New York, USA.

\begin{abstract}
Signal transducer and activator of transcription 3 (Stat3) is a transcription factor that is constitutively activated in a variety of human malignancies, including prostate, lung, brain, breast, and squamous cell carcinomas. Inhibition of activated Stat 3 leads to decreased proliferation and apoptosis of many cancerderived cell lines, while the introduction of a constitutively activated form of Stat 3 into immortalized human breast epithelial cells and rodent fibroblasts results in cellular transformation. Collectively, these data suggest a role for Stat 3 in oncogenesis. A new study from Chan et al. (see related article beginning on page 720 ) is the first to demonstrate a requirement for Stat3 in de novo epithelial carcinogenesis in vivo. Using the two-step model of chemically induced skin carcinogenesis, the authors demonstrated that mice deficient in Stat 3 were completely resistant to skin tumor development.
\end{abstract}

Stat's (signal transducers and activators of transcription) are a family of latent transcription factors that are activated in response to many cytokines and growth factors. Stat activation is dependent upon tyrosine phosphorylation, which induces dimerization via reciprocal phosphotyrosine-src homology domain 2 (phosphotyrosine-SH2) interaction between two Stat molecules. Activated Stat's translocate to the nucleus where they bind to consensus promoter sequences of target genes and activate their transcription (1) (Figure 1). Many tyrosine kinases, including JAKs (Janus kinases), RTKs (receptor tyrosine kinases), and non-RTKs can phosphorylate Stat proteins. In normal cells, Stat tyrosine phosphorylation is transient, lasting from 30 minutes to several hours.

Nonstandard abbreviations used: DMBA, 7,12dimethylbenz[a]anthracene; Ha-ras, Harvey rat sarcoma virus oncogene; JAK, Janus kinase; LRC, label-retaining cell; RTK, receptor tyrosine kinase; SH2, src homology domain 2; Stat3, signal transducer and activator of transcription 3; TPA, 12-O-tetradecanoylphorbol-13-acetate; v-Ha-ras, Ha-ras homolog.

Conflict of interest: The authors have declared that no conflict of interest exists.

Citation for this article: J. Clin. Invest. 114:619-622 (2004). doi:10.1172/JCI200422800.
However, in numerous cancer-derived cell lines or in primary tumors, Stat proteins (in particular Stat3) are persistently tyrosine phosphorylated either as a consequence of deregulated positive effectors of Stat activation such as tyrosine kinases or negative regulators of Stat phosphorylation, e.g., phosphatases, suppressor of cytokine signaling, protein inhibitor of activated stats) (2). Inhibition of Stat 3 activity in tumor-derived cell lines by the introduction of antisense, small interfering RNA, dominant-negative Stat 3 constructs, and/or blockade of tyrosine kinases has been associated with growth arrest and apoptosis (2). Furthermore, the introduction of a constitutively activated Stat 3 molecule (Stat3C) into immortalized cell lines leads to transformation, indicating an oncogenic role for activated Stat $3(3,4)$. A possible mechanism for transformation by activated Stat 3 is the transcriptional upregulation of genes known to be involved in proliferation and apoptosis, including Bcl-xL, c-Myc, cyclin D1, Vegf, and Survivin (3, $5-8)$. In addition to its role as a transcription factor, phosphorylated Stat 3 has been described in a recent report as a component of focal adhesions (sites of cell contact with the extracellular matrix) that may contribute to the invasiveness of ovarian cancer cells (9). The in vivo role of Stat 3 in tumorigenesis has not been addressed until now. In this issue of the JCI, Chan and colleagues demonstrate in two different murine models of skin tumor development that Stat 3 is required for de novo tumorigenesis (10).

One of the best-established model systems for studying the mechanisms underlying the process of malignant transformation is the mouse skin model of multistage carcinogenesis $(11,12)$ (Figure 2). In this model, the process of skin tumor development can be subdivided into three different stages: initiation, promotion, and progression. Initiation is typically induced by the topical application of the carcinogen 7,12-dimethylbenz[a]anthracene (DMBA). Interestingly, in DMBA-treated epidermal cells, one usually finds mutations within the Harvey rat sarcoma virus oncogene (Ha-ras) gene. These mutations, however, are not sufficient to induce de novo transformation. Promotion of tumorigenesis is generated by the topical application of phorbol esters such as 12-O-tetradecanoylphorbol-13-acetate (TPA) to the skin, leading to epithelial cell proliferation with a concomitant increased expression of the ligand EGF as well as of cyclin D1, c-Jun, c-Fos, and c-Myc (13-16). TPA-treated mice form multiple benign papillomas within 10-20 weeks. Tumor progression is a spontaneous process resulting in the formation of malignant squamous carcinomas.

\section{Stat3 prevents apoptosis in the initiation phase of skin tumorigenesis}

K5Cre.Stat $3^{\mathrm{fl} / \mathrm{fl}}$ transgenic mice, whose epidermal and follicular keratinocytes lack Stat 3 , are viable and develop normally. 